\title{
An unusual transmission event of Neisseria meningitidis serogroup W135 type 2a in a healthcare setting, England, 2012
}

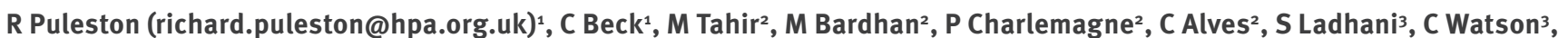

M Ramsay ${ }^{3}$, E Kaczmarksi4, R Borrow ${ }^{4}$, S Gray ${ }^{4}$, D Hadlington ${ }^{5}$, M Weinbren $^{6}$, D Bhattacharjee ${ }^{6}$, N Inglis

1. Health Protection Agency, Nottingham, United Kingdom

2. Health Protection Agency, Birmingham, United Kingdom

3. Health Protection Agency, London, United Kingdom

4. Meningococcal Reference Unit, Health Protection Agency, Public Health Laboratory, Manchester, United Kingdom

5. West Midlands Ambulance Service, West Midlands, United Kingdom

6. University Hospitals Coventry and Warwickshire NHS Trust, Coventry, United Kingdom

7. NHS Coventry and Warwickshire, Coventry, United Kingdom

Citation style for this article:

Puleston R, Beck C, Tahir M, Bardhan M, Charlemagne P, Alves C, Ladhani S, Watson C, Ramsay M, Kaczmarksi E, Borrow R, Gray S, Hadlington D, Weinbren M,

Bhattacharjee D, Inglis N. An unusual transmission event of Neisseria meningitidis serogroup W135 type $2 a$ in a healthcare setting, England, 2012 . Euro Surveill.

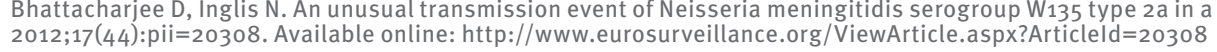

Article submitted on 08 October 2012 / published on 01 November 2012

We report an outbreak of Neisseria meningitidis serogroup $\mathrm{W}_{135}$, associated with a transient transmission event between asymptomatic individuals in a healthcare setting. Two elderly persons subsequently developed invasive meningococcal disease. The duration and type of close contact for those directly involved in the probable transmission incident would not have warranted chemoprophylaxis according to current guidelines. Meningococcal infection in older persons usually presents with pneumonia rather than meningitis or septicaemia with purpura.

We report a nosocomial outbreak of Neisseria meningitidis serogroup $\mathrm{W}_{135}$, phenotype 2a:P1.5, 2 associated with a transient transmission event between asymptomatic individuals in March 2012. Two elderly individuals admitted to the same hospital group (at different sites) presented within four days of each other with non-specific respiratory tract symptoms of infection. Initial microbiological analysis isolated N. meningitidis serogroup $\mathrm{W}_{135}: 2 \mathrm{a}: \mathrm{P}_{1}$.5, 2 (porA genotype 5, 2, 36-2) from both patients' blood cultures. The identical microbiological phenotype/genotype of these isolates suggested an epidemiological link.

\section{Epidemiological investigation}

The epidemiological investigation revealed that both patients had in the preceding weeks been admitted to different wards in the same hospital for unrelated illnesses, but had not been in contact with each other during that stay (admitted 19 and 16 days before the positive blood culture result, with stays of 10 and four days, respectively, for the initial admission). However, on the day of discharge, both were transported in the same hospital vehicle at the same time, one to a rehabilitation facility and the other to a care home. Both became ill with non-specific symptoms (however, respiratory infection was thought to be the most likely cause) within six and 10 days after transport, respectively. One patient remained at the rehabilitation unit for management of the disease and the other was readmitted to hospital for treatment. Since the most likely epidemiological link between the two cases was the short transfer journey (duration 67 minutes), nasopharyngeal swabs were obtained from the two staff members involved in the transport and another passenger who was on the vehicle at the same time as the cases, but for less than half an hour.

One of the transport staff (in their 6os) was also found to be carrying serogroup $\mathrm{W}_{135}: 2 \mathrm{a}: \mathrm{P}_{1.5}, 2$ (porA 5, 2, 36-2) and reported having had upper respiratory tract symptoms in the intervening period (symptom onset 16 days after the patient transport), but had not sought medical attention. The swabs from the second transport team member and the other passenger were negative. None of the affected individuals had travelled abroad during the incubation period. It was not possible to identify the primary case or the sequence of transmission events. For this reason chemoprophylaxis was offered to all staff and patients who had been in close contact with the two infected patients during the incubation period (in the hospital, the transport vehicle and the community) up until 24 hours after commencement of antibiotic treatment of the cases. Chemoprophylaxis and meningococcal $\mathrm{ACW}_{135} \mathrm{Y}$ quadrivalent conjugate vaccination was also arranged for the swab-positive transport staff member and their close contacts.

Subsequent multilocus sequence typing of the samples obtained from this incident confirmed the epidemiological link between the affected individuals. All were determined to have the same strain designation W135:2a:P1.5,2:F1-1:ST-11 (cc11). 
Laboratory-confirmed W135 Neisseria meningitidis cases, deaths, and case fatality ratio by age group, England and Wales, 2006-12 $(\mathrm{n}=162)$

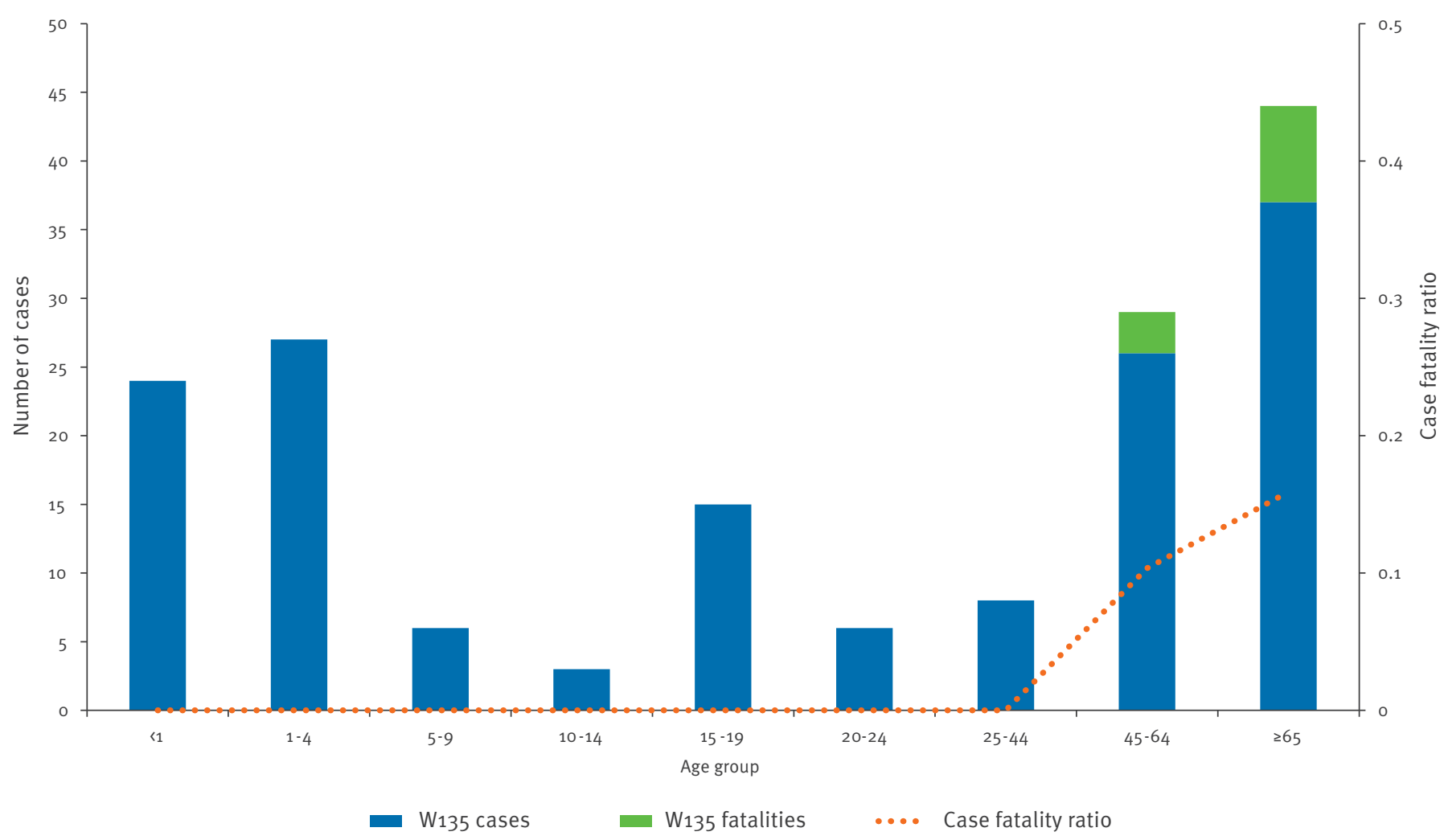

Source: Surveillance data, Health Protection Agency.

\section{Background information}

In England and Wales, invasive meningococcal disease most commonly occurs in children under five years of age and teenagers (between the years 2006 and 2011 at incidences of $38.6 / 100,000$ for the under one yearolds, $12.6 / 100,000$ in children aged one to four years, and 3.2/100,000 for teenagers aged from 15 to 19 years) $[1,2]$. N. meningitidis is transmitted through direct contact with infectious respiratory secretions, droplets or aerosols. Typically, for transmission to occur, close, prolonged contact (typically accepted as eight hours or more) is required $[1,3-5]$. Cases associated with public transport have been noted, but they involved extended contact over a period of hours [6].

Since the meningococcal serogroup $C$ conjugate vaccine was introduced in 1999 to the childhood immunisation schedule in the United Kingdom (UK), around 85-90\% of invasive disease cases have been due to N. meningitidis serogroup B [2]. Invasive disease by the $\mathrm{W}_{135}$ serogroup is infrequently seen. For example, between 2006 and 2011, serogroup W135 accounted for only $2.3 \%$ of cases (annual average: 26 W135 cases) of laboratory-confirmed meningococcal disease in England and Wales, and most occurred in the very young or the very old [2]. Fatalities occurred in $5.5 \%$ of these cases, all in adults older than 45 years [2] (Figure)

\section{Discussion and conclusions}

In the last 10 years, most $W_{135}$ organisms isolated in the UK were typed as W135: NT: P1.3.6 cc22 or combinations thereof. In contrast, as reported in this incident, W135:2a:P1.5, 2 cc11 has been only infrequently observed: 32 cases from 2009 to May 2012 (personal communication Dr S Gray, May 2012).

The occurrence described here of invasive meningococcal disease with serogroup W135 may have resulted from a single transient period of close contact. It is notable since symptoms suggestive of meningococcal infection were not apparent at that point. Despite detailed epidemiological and microbiological analysis, it was not possible to determine the sequence of transmission.

Like serogroup $\mathrm{Y}$, most serogroup $\mathrm{W}_{135}$ infections in older adults present as pneumonia (usually in the presence of comorbidities) and are invariably confirmed following a positive blood culture [2,7-9]. Public health notification on clinical suspicion of meningococcal 
disease is unusual in elderly persons. Where cases are notified in this age group, they are more likely to be caused by the less prevalent serogroups $[2,10]$.

Epidemiological investigations of clonal complex 11 (cc11) N. meningitidis serogroup C have previously demonstrated low levels of carriage and increased case fatality ratios when compared to other clonal complexes [11-14]. It is not known if $N$. meningitidis expressing the serogroup W135 capsular polysaccharide and belonging to cc11 have similar epidemiological features (such as duration of carriage) as serogroup $C$ ST11 organisms. Nonetheless, previous findings from individuals returning from the Hajj demonstrate the capacity of the W135 strain associated with the pilgrimage to cause infection in contacts up to 36 days post return [15].

This cluster of epidemiologically linked serogroup W135 infections raises important implications for the public health and clinical management of the disease, particularly in older age groups. Occupational transmission of meningococcal disease has been previously observed, mostly in laboratory settings. Instances in which emergency workers have been infected have been seen although only from clearly symptomatic patients [16-18].

Antibiotic treatment for cases and prophylaxis for their contacts to eradicate carriage and therefore reduce local transmission is warranted. In addition, for contacts of cases with serogroups ACWY, conjugate vaccines against the specific serogroup may further protect contacts and reduce carriage and local transmission $[15,19]$. Clinicians need to be aware that while $N$. meningitidis invasive disease is most common in young children and adolescents, it can occur in the elderly, although the presentation will probably differ [2]. Respiratory symptoms predominate in the elderly rather than clinically evident meningitis or septicaemia with purpura. Invasive meningococcal disease caused by all serogroups has a substantially higher case fatality rate in the elderly than in younger age groups. Comorbidities are recognised as an associated risk factor and may explain the poorer outcome [2].

Cases of invasive meningococcal disease occurring in older persons should receive equally robust public health investigation and management as those in younger individuals. Although the duration and type of close contact for the individuals exposed in the ambulance as described here would not have fulfilled the criteria for chemoprophylaxis according to current national public health guidelines, the common epidemiological links allowed appropriate control measures to be implemented. Defining close contacts in incidents where a healthcare source is suspected may be problematic technically and also may provoke anxiety for those involved. A flexible approach utilising expert public health advice to guide intervention is therefore appropriate for this type of occurrence [5].
References

1. Meningococcal meningitis and septicaemia notifiable. In: Salisbury D, Ramsay M, Noakes K, editors. Immunisation against infectious disease. The Green Book. London: Department of Health; 2007. p. 235-55.

2. Ladhani SN, Flood JS, Ramsay ME, Campbell H, Gray SJ, Kaczmarski EB, et al. Invasive meningococcal disease in England and Wales: Implications for the introduction of new vaccines. Vaccine. 2012;30(24):3710-6.

3. Meningococcal Disease. In: Atkinson W, Wolfe C, Hamborsky J, editors. Epidemiology and Prevention of Vaccine-Preventable Diseases The Pink Book. 12th ed. Washington D.C.: Centers for Disease Control and Prevention, The Public Health Foundation; 2012. p. 203.

4. Caugant DA, Maiden MCJ. Meningococcal carriage and diseasepopulation biology and evolution. Vaccine. 2009;27 Suppl 2:B64-70.

5. Health Protection Agency (HPA) Meningococcus and Haemophilus Forum. Guidance for public health management of meningococcal disease in the UK. London: HPA; 2012. Available from: http://www.hpa.org.uk/webc/HPAwebFile/ HPAweb_C/1194947389261

6. Mohr O, Askar M, Schink S, Eckmanns T, Krause G, Poggensee G. Evidence for airborne infectious disease transmission in public ground transport - a literature review. Eurosurveillance. 2012;17(35): pii=20255. Available from: http://www. eurosurveillance.org/ViewArticle.aspx?Articleld=20255

7. Hershey JH, Hitchcock W. Epidemiology and meningococcal serogroup distribution in the United States. Clinical Pediatrics (Phila). 2010;49(6):519-24.

8. Ladhani SN, Lucidarme J, Newbold LS, Gray SJ, Carr AD, Findlow J, et al. Invasive meningococcal capsular group $Y$ disease, England and Wales, 2007-2009. Emerg Infect Dis. 2012;18(1):63-70.

9. Rosenstein NE, Perkins BA, Stephens DS, Lefkowitz L, Cartter ML, Danila R, et al. The changing epidemiology of meningococcal disease in the United States, 1992-1996. J Infect Dis. 1999;180(6):1894-901.

10. Harrison LH. Epidemiological profile of meningococcal disease in the United States. Clin Infect Dis. 2010;50 Suppl 2:S37-44.

11. Trotter CL, Fox AJ, Ramsay ME, Sadler F, Gray SJ, Mallard $R$, et al. Fatal outcome from meningococcal disease - an association with meningococcal phenotype but not with reduced susceptibility to benzylpenicillin. J Med Microbiol. 2002;51(10):855-60.

12. Buckee CO, Jolley KA, Recker M, Penman B, Kriz P, Gupta S, et al. Role of selection in the emergence of lineages and the evolution of virulence in Neisseria meningitidis. Proc Natl Acad Sci U.S.A. 2008;105(39):15082-7.

13. Ibarz-Pavón AB, Maclennan J, Andrews NJ, Gray SJ, Urwin $\mathrm{R}$, Clarke SC, et al. Changes in serogroup and genotype prevalence among carried meningococci in the United Kingdom during vaccine implementation. J Infect Dis. 2011;204(7):1046-53.

14. Maiden MCI, Stuart JM. Carriage of serogroup C meningococci 1 year after meningococcal $C$ conjugate polysaccharide vaccination. Lancet. 2002;359(9320):1829-31.

15. Wilder-Smith A, Goh KT, Barkham T, Paton NI. Hajj-associated outbreak strain of Neisseria meningitidis serogroup W135: estimates of the attack rate in a defined population and the risk of invasive disease developing in carriers. Clin Infect Dis. 2003;36(6):679-83.

16. Baron EJ, Miller JM. Bacterial and fungal infections among diagnostic laboratory workers: evaluating the risks. Diagn Microbiol Infect Dis. 2008;60(3):241-6.

17. Centers for Disease Control and Prevention (CDC). Occupational Transmission of Neisseria meningitidis - California, 2009. MMWR Morb Mortal Wkly Rep. 2010;59(45):1480-3.

18. Sejvar JJ, Johnson D, Popovic T, Miller JM, Downes F, Somsel P, et al. Assessing the risk of laboratory-acquired meningococcal disease. J Clin Microbiol. 2005;43(9):4811-4.

19. Wilder-Smith A, Chow A, Goh KT. Emergence and disappearance of W135 meningococcal disease. Epidemiol Infect. 2010;138(7):976-8. 